\title{
Scope and Challenge of Computational Methods for Studying Mechanism and Reactivity in
}

\section{Homogeneous Catalysis}

Jeremy N. Harvey, ${ }_{\dagger}^{*}$ Fahmi Himo, $*_{+}^{*}$ Feliu Maseras $*_{g}$ and Lionel Perrin ${ }_{+\dagger}^{+\dagger}$

$\dagger$ Department of Chemistry, KU Leuven, Celestijnenlaan 200F, B-3001 Leuven, Belgium.

† Department of Organic Chemistry, Arrhenius Laboratory, Stockholm University, SE-106 91 Stockholm, Sweden.

I Institute of Chemical Research of Catalonia (ICIQ), The Barcelona Institute of Science and Technology, Avgda. Països Catalans, 16, 43007 Tarragona, Catalonia, Spain.

$\dagger \dagger$ Univ Lyon, Université Claude Bernard Lyon 1, CPE Lyon, INSA Lyon, ICBMS, CNRS UMR 5246, 43 Bd. du 11 Novembre 1918, 69622 Villeurbanne, France. 
ToC Graphic:

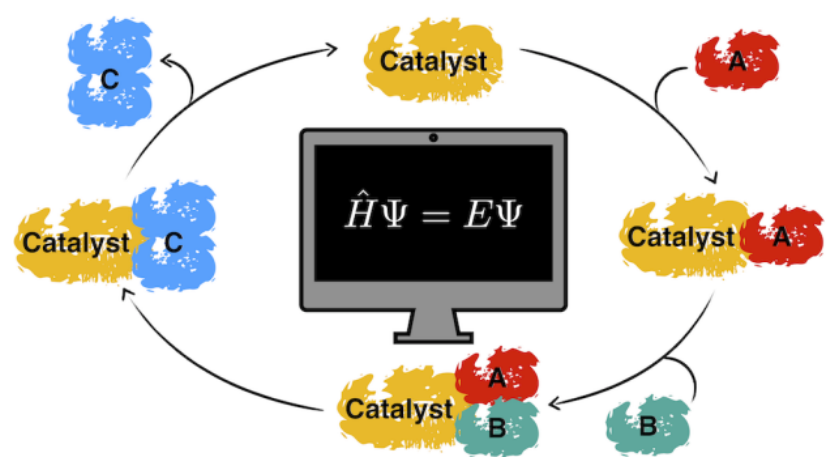

ABSTRACT. Computational methods based on quantum mechanical modelling are increasingly used to provide insight into mechanistic aspects of homogeneous catalysis. While the potential and value of such methods is obvious, it is also clear that it remains challenging to obtain reliable and predictive mechanistic insights from modelling. In this perspective, we assess the various factors influencing the quality of computational studies. While the type of electronic structure theory methodology used is of course of great importance, we argue that many other aspects can play a large role also. The other factors emphasized here include the treatment of entropic effects, solvation, the choice of the structural model, conformational complexity, the translation of computed relative Gibbs energies into a kinetic model, and the high demands required for the prediction of selectivity.

KEYWORDS. Homogeneous catalysis, computational chemistry, entropy, solvation, conformers, kinetics, selectivity.

\section{Introduction}


Computational methods based on first-principles studies of electronic structure have made tremendous progress in the last decades due to faster computers, more efficient algorithms and more accurate methods. As a result, mechanistic studies of catalysis rely much more on computation than was previously the case. As well as providing additional insight into the nature of intermediates and transition states whose existence has been suggested by kinetic or spectroscopic studies, theory is increasingly expected to provide quantitative predictions of mechanisms. Indeed, going beyond just predicting mechanism, theory is often now expected to be able to be used as an underlying tool for designing improved catalysts.

This hugely enhanced role is a powerful testimony to the increased power of computation, but it should also be recognized that it brings with it some problems. Deriving a full mechanistic picture based only on computation remains highly challenging, and here and there, one encounters arguments whereby theory cannot be relied upon for this purpose. Two prominent examples can be given: In a recent editorial,1 Noyori and Richmond argued that "[R]esearchers often overly rely on computation in interpreting a reaction mechanism. The conclusions $[\ldots]$ are far from truth, and confuse and mislead the community. [...] We should appreciate solid experimental evidence more than frivolous computations. This prevailing trend is harmful to the community." Elsewhere, in a discussion of the contribution of computational studies to the mechanism of an organic reaction, Plata and Singleton2 stated that "In the terminology of Pauli, computational mechanistic chemistry is "not even wrong" about the [discussed] mechanism." We do not intend to litigate these arguments here: the consensus among the present authors as well as those just cited is that computation is remarkably helpful in approaching catalytic reaction mechanisms, even though it also frequently leads to incorrect predictions. Rather, we intend to sketch some thoughts about best 
practices in the field emerging from our own experiences as well as those of others, with the aim that the proportion of successes should increase.

Quite obviously, improved accuracy of computational mechanistic studies relies on using state of the art methodology. This truism is frequently taken to mean that one should use the best possible form of electronic structure theory - indeed, the apparently general phrase "level of theory" is often taken in a restrictive sense to refer only to the type of electronic structure theory method used. We do not seek to deny the importance of this aspect, nor the major impact of progress that has taken place in this area in recent decades, among which one can mention the development of accurate density functional theory approaches (including improved treatment of dispersion3), of efficient methods to treat the electron-electron interaction explicitly within correlated techniques, 4 of techniques for treating electron correlation locally so as to improve the scaling of computational methods, 5 and the exploitation of novel computer architectures to obtain faster codes.6 However, many other aspects play an important role also, and have received less attention. In our view, some of the key factors to take into account when studying the mechanisms of homogeneous catalysis are (Scheme 1), as well as the choice of electronic structure methodology: the treatment of entropy and other thermal effects; the treatment of solvation; the choice of the microscopic or structural model used to represent the target chemistry; the treatment of conformational complexity; the model used to predict rate constants for elementary steps in the mechanism; how the rates and rate constants of elementary steps combine to give rise to overall kinetics; the challenges associated with predicting selectivity, and other aspects such as modelling of electron-transfer steps and dynamics. 


\section{Accuracy in Theoretical \\ Homogeneous Catalysis}

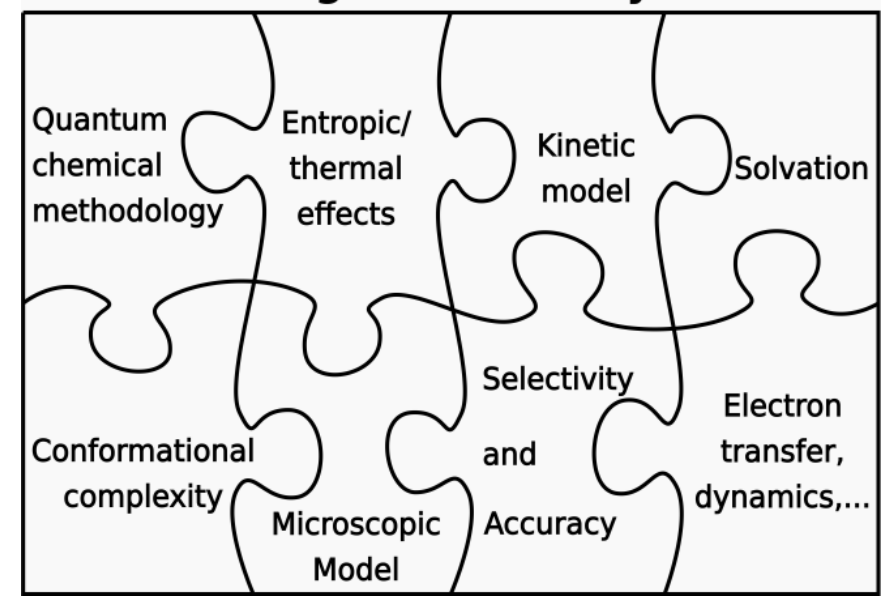

Scheme 1.

In this article, we will discuss each of these aspects relating to methodology and attempt to summarize the state of the art for each of them, drawing upon illustrative examples from our own work and that of others. We should point out that this article is primarily concerned with a discussion of the methods, and in no way intends to summarize the many achievements in the field, which have been previously reviewed elsewhere, including in many cases discussion of the challenges in a way that overlaps with the present work (For some examples, see refs. $7,8,9,10,11,12,13,14,15)$.

\section{Electronic Structure Methodology}

We start by briefly reviewing electronic structure methodology. This is an area where enormous progress has been made in the last decades, which is perhaps best measured by comparing to past methodology for modelling catalytic reaction mechanisms. In an early study of the transition state for hydrogen oxidative addition to $\mathrm{Pt}(0)$, Kitaura, Obara and Morokuma could only carry out geometry optimization with Hartree-Fock theory and a very modest basis set, with some limited configuration interaction treatment of correlation applied in single-point calculations.16 Almost four decades later, we can see that a major point of progress was the development of density 
functional theory, with its combination of relative affordability and reasonable accuracy (especially after the importance of dispersion was acknowledged). A second breakthrough that is finally delivering on its formidable promise 17 thanks to the dedicated work of many groups is the ability to treat electron correlation effects rigorously, accurately and affordably using local correlation methods.5 The tantalizing prospect of being able to obtain potential energies with 'chemical' accuracy of better than $1 \mathrm{kcal}$ mol-1 on a routine basis seems to have drawn much closer in recent years.

\section{Entropy and Thermal Effects}

It is well known that key mechanistic observed properties do not depend directly on electronic energies, but instead on free energies such as Gibbs or Helmholtz energies. The electronic energy is a key ingredient in theoretical expressions for the Gibbs energy, but the other terms, associated with thermal excitation at a given finite temperature, and most importantly with entropic effects, also play an important role. A useful rule of thumb18 well-known to many computational chemists gives a sense of the importance of these effects: for any reaction step in which two molecules combine to form a single one, the standard Gibbs energy change at room temperature will be roughly $10 \mathrm{kcal}$ mol-1 less favourable (or more unfavourable) than the electronic energy change. When considering catalytic reaction mechanisms, it is far from rare to need to compare Gibbs energies for states of the system in which the molecularity differs by more than one, so that entropic contributions $T \Delta S$ equal to multiples of $10 \mathrm{kcal} \mathrm{mol-1}$ intervene. This is not a small effect!

Rigorous calculation of Gibbs energies from first principles is not straightforward, since it requires access to the partition function of the system. In principle, to calculate this accurately for the large solvated molecular systems that are important in homogeneous catalysis, one needs to 
sample a broad range of structures of the solute but also of the solvent, while using an accurate method for computing the potential energy. While approaches based on explicit sampling are sometimes used in the context of computational studies of homogeneous catalysis, 19,20 the most common way to calculate Gibbs energies is to use techniques based on treating different types of degrees of freedom separately, and with separate approximations. Vibrational frequencies are mostly treated using the harmonic oscillator approximation, sometimes with simple corrections to take into account anharmonic effects for soft degrees of freedom such as hindered rotors, 21,22 and indeed sometimes with more rigorous anharmonic treatments.23 Rotational (or librational) degrees of freedom are treated using ideal gas expressions, and translational degrees of freedom are treated with the Sackur-Tetrode equation, again an expression that is strictly speaking applicable only to ideal gases. Note that for results relating to solution-phase experiments, the output from many quantum-chemistry codes needs to be corrected, as they usually assume a gas-phase standard state when computing the translational entropy. For gases, it is natural to use a standard state corresponding to an ideal gas at $p_{0}=1$ bar when computing the available volume in the SackurTetrode equation, but for solutions, a standard state corresponding to $[\mathrm{X}]_{0}=1 \mathrm{M}$ yields a different value; the switch from one standard state to the other is easily performed by adding a correction term $R T \ln V_{\mathrm{m} 0}=1.89 \mathrm{kcal} \mathrm{mol}-1$ at $298 \mathrm{~K}$ (derived from the molar volume of ideal gases at $p_{0}$ ).

The use of the Sackur-Tetrode equation, the rigid rotor approximation and other ideal gas-based formulas to compute entropies in solution has been much debated in recent years. In our view, this debate has arisen due to three dominant reasons. First, the large magnitude of entropy corrections for reaction steps involving a change in molecularity means that they frequently lead to a major change in the qualitative energy surface emerging from quantum chemical studies. In many cases, this correction leads to a degradation in the level of agreement with experiment when the 
underlying quantum chemical method is a DFT functional that neglects dispersion effects or describes them poorly, such as most of the functionals that were heavily used in the 90's and 00's for study of homogeneous catalysis. This is because the entropy correction destabilizes aggregates of multiple fragments, and so does the neglect of dispersion attractive forces. The net effect is that for apparently "well understood" systems, adding the ideal gas-like entropy correction appeared to lead to incorrect results.

The second reason is that the intuitive picture one has of rotational or librational motion of molecules in solution, and of their translational motion, is severely at odds with the ideal gas picture of free rotation and translation. In solution, molecules can of course rotate and translate, but they do so in a diffusional manner involving frequent collisions with solvent molecules, in a way that is very different from gaseous molecules. Viewed from the correct statistical mechanics perspective, this difference is not necessarily relevant. The distribution of kinetic energies for the solute atoms will still be roughly the same as it would be for the same molecules in the gas phase at a given temperature, and the solute will still sample all orientations and all positions within the available volume, so both the kinetic and configurational contributions to the partition function will in this sense not be changed. Nevertheless, intuitively, there seems to be a strong appeal to the idea that the solute's entropy should be lower.

The third factor is that where entropies of solvation are known from experiment, they are usually quite negative, 24 suggesting that molecules undergo a loss of disorder upon solvation, that must be reflected in the theoretical treatment. Part of the reason for the negative entropies of solvation comes from the fact that the standard state used for expressing the thermodynamic functions is often different in the gas phase (where it is usually $p_{0}=1 \mathrm{bar}$ ) and in solution (where it is more often $\left.[\mathrm{X}]_{0}=1 \mathrm{~mol} \mathrm{dm}-3\right)$, but even when this is taken into account, the entropy of solvation is often 
quite strongly negative. Viewed from the correct statistical mechanical point of view, this is not due to the intuitive 'hindered-rotation-and-translational' model described above, but must instead be associated with reduced conformational freedom of the solute-plus-solvent system associated with structural perturbation of the solvent cage. Still, intuitively, it is tempting to attribute it instead to reduced mobility of the solute when in solvent.

The result of these factors is that the use of modified approximate methods for calculating entropy corrections in solution has become widespread. The simplest such modified approaches are based on applying a uniform scaling factor to the entropy term calculated using ideal gas expressions.25 Another commonly used formalism uses instead a modified "available volume" in the Sackur-Tetrode equation.26,27 In this approach, instead of using the full volume corresponding to the relevant standard state to evaluate the translational partition function, a reduced volume corresponding to the available space within the solvent is used. In practice, this leads to a very similar effect to the scaling approach, with entropy effects being significantly reduced compared to those obtained when using the unmodified ideal gas expressions.

Given that solvation entropies are indeed quite often negative, when evaluating solution-phase entropies ab initio, some sort of correction compared to the ideal gas expressions is indeed necessary. However, in practice, this correction is almost universally applied through another part of the computational protocol, namely the use of a continuum model of solvation. In most quantum-chemical studies of solution-phase homogeneous catalysis, especially those involving polar species in polar solvents, such models are applied either as single-point energy corrections for structures optimized in vacuum, or self-consistently throughout geometry optimization. Either way, such models lead to computed "potential energies" that are in fact hybrid quantities taking into account - albeit approximately - the Gibbs energy contributions arising from integrating over 
all possible solvent structures surrounding the solute. As such, they include by construction the (often negative) solvation entropy terms that are mentioned above. Hence including a second correction for these terms is not correct. Consider the example of dissolving methane in watersimilar to the rare gas examples used as motivation for the available volume model. Experimentally, the corresponding Gibbs energy of solvation is $+2 \mathrm{kcal}$ mol-1,22 likely reflecting some combination of a weakly negative enthalpy of solvation associated with solvent reorganization and solute-solvent interactions, and a quite strongly negative entropy of solvation due to solvent reorganization. The SMD continuum solvent model returns a calculated Gibbs energy of solvation (at the B3LYP/6-31G(d) level of theory) of $+2.3 \mathrm{kcal}$ mol-1, very close to the experimental value. In this way, any calculation treating methane in water when using a continuum model will account for its negative solvation entropy.

We therefore strongly argue that the best currently available way to handle entropic effects for molecules or transition states in solution is through the usual ideal-gas expressions, combined with continuum solvent treatment of solvation Gibbs energy, and careful attention to the use of the correct reference standard state. Where needed, attention should be paid to the subtle effects on structure or on vibrational frequencies upon solvation, so that partition functions should be calculated based on solute properties computed with continuum solvent, rather than in vacuum. In practice, though, this usually only leads to small differences.28 Also, attention should be paid to the possible shortcomings of the continuum models, particularly for strongly solvated, charged or highly polar species. But the correction terms mentioned above should not be included. Indeed, multiple examples from our own groups in recent years reach good agreement between calculated and experimental Gibbs energies based on including 'full' ideal-gas-like entropy corrections for 
system in solution, where the magnitude of the entropy effects is so large that any scaling or related correction would lead to large changes in computed relative Gibbs energies.19,29

\section{Solvation Effects}

When studying reaction mechanisms in solution using computational methods, it is usually very important to include a good description of solvation effects. The basic physical understanding of solvent effects should be familiar to most quantum chemists, with the dominant effect being the differential strength of interactions between solvent molecules and neutral, dipolar, zwitterionic and ionic species.30 Hence in any reaction where the chemical species involved undergo significant changes in polarity or more generally changes in the ratio of the local charge and the local radius for solvent-exposed parts of the molecule, differences between calculated gas phase and solution phase Gibbs energies must be expected. However, it is easy to forget just how large this effect is. In the gas phase, typical anions have proton affinities of over $200 \mathrm{kcal} \mathrm{mol-1,} \mathrm{yet} \mathrm{this} \mathrm{may} \mathrm{map}$ onto a Gibbs energy difference for acid dissociation of the corresponding neutral species in solution that is close to zero. Formation of zwitterionic or simply highly polar intermediates from neutral or less polar species can also lead to solvation Gibbs energy effects of over 20 kcal mol-1.

The most common way to treat these huge solvation effects in quantum chemical studies is to use a continuum model.31 Such models apply the bulk equations of electrostatics to the solvent, with a self-consistent interface to the solute treated in the quantum chemical calculation, in the

form of Coulomb's Law electrostatic interactions between the solute charge distribution and a distribution of charges forming a model of the polarized solvent. The models are carefully developed to describe the physics of solvation correctly, and the parameters in the models are calibrated to reproduce known experimental data for solvation of both neutral species and ions. There are many different approaches to continuum solvent modelling, and many different 
implementations in quantum chemical packages - too many to be discussed in detail here. Relative accuracy of the different approaches is much discussed in the literature, and this aspect will not be reviewed here. Instead we will simply point out that the average accuracy of all these models is impressive: for many solvents, mean unsigned errors on solvation Gibbs energies for neutral

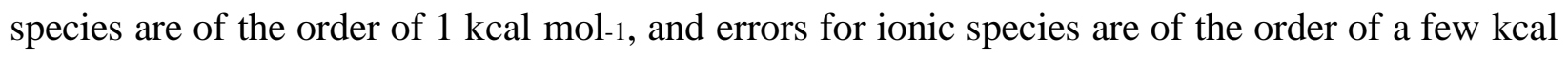
mol-1.22 Considering the microscopic complexity of the solvation process, and the challenge in reaching kcal mol-1 accuracy for quantities such as bond energies or heats of formation with quantum chemistry, this outcome is remarkable. Also, as often in quantum-chemical modelling, disagreement between different models can be taken as an informal indication of the likely error bars associated with the continuum approach.

Nevertheless, two limitations still need to be borne in mind. First, continuum solvent models are parameterized to reproduce solvation Gibbs energies. The result of a quantum chemical calculation with an arbitrary solute structure is a hybrid quantity, having some of the properties of the potential energies familiar from quantum chemical calculations in vacuum, and some properties of a Gibbs energy. It resembles a potential energy in that it changes with solute structure, and has minima, saddle-points, etc. in the space of solute coordinates. Yet by referring to the average effect of solvent molecules at a given temperature, it resembles a Gibbs energy. In these senses it is a potential of mean force obtained by (implicitly) integrating a Boltzmann factor over all possible solvent coordinates while maintaining a given solute structure (for a clear discussion of this aspect, see ref. 28). Given this definition, there are some limitations on how energies resulting from continuum models can be used. For example, it means that it is difficult to use such models to compute reaction or activation enthalpies in solution. One might be tempted to estimate such enthalpies by using rigid-rotor, harmonic oscillator, particle-in-a-box statistical mechanics for the 
solute, combined with the continuum model to 'treat solvent effects'. This could be done either by including the continuum solvent effects in the necessary geometry optimization and frequency calculation steps, or by performing these in vacuum, then computing a single-point calculation of the solvent effect.32 Either way, what one will obtain is not an enthalpy, since the solvation term is a Gibbs energy.

To illustrate this problem, consider an ionic dissociation reaction $\mathrm{A} \rightarrow \mathrm{B}++\mathrm{C}_{-}$, associated with an enthalpy change $\Delta H_{\mathrm{g}}$ in vacuum. Using the above protocol will yield a quantity $\Delta H_{\mathrm{g}}+\Delta \Delta H_{\text {solv }}$ $-T \Delta \Delta S_{\text {solv }}$, where $\Delta \Delta H_{\text {solv }}$ and $\Delta \Delta S_{\text {solv }}$ are the difference in enthalpy and entropy of solvation of the reactants and products. The associated change in Gibbs energy of solvation $\Delta \Delta G_{\text {solv }}$ will be very negative, reflecting the better solvation of the ionic species, and this will in most cases map on to an even more negative $\Delta \Delta H_{\text {solv }}$ but a positive entropy contribution $-T \Delta \Delta S_{\text {solv }}$, due to enthalpically favourable but entropically costly arrangement of the solvent around the ions. Comparing the $\Delta H_{\mathrm{g}}+\Delta \Delta H_{\text {solv }}-T \Delta \Delta S_{\text {solv }}$ to the experimental $\Delta H_{\mathrm{g}}+\Delta \Delta H_{\text {solv }}$ will yield agreement only if the solvation entropic contribution is very small, or due to cancellation with some other error.

In principle, continuum solvent models can be parameterized to return separate enthalpies and entropies of solvation, and indeed, this could be done at multiple different temperatures. The amount of experimental data available for doing such fitting is however rather inadequate for most solvents. Also, the parameterization process for continuum models is already complex enough without such enhancements. While some studies of homogeneous catalysis have used continuum approaches to derive separate enthalpies and entropies, 33 in practice, for most applications, continuum models are 'limited' to yielding Gibbs energies of solvation at $298 \mathrm{~K}$. Where separate 
enthalpies or entropies are needed, or where solvation Gibbs energies at temperatures very different from $298 \mathrm{~K}$ are needed, then the continuum approach will not be sufficient.

A second limitation relates to highly polar species. Species with a high local charge to local radius ratio (such as small ions, or larger ions, or polar species where there is a large build-up of charge on a small number of small, solvent-exposed, atoms) can interact very strongly with solvent, e.g. by accepting a hydrogen bond from solvents such as water or alcohols, or by donating hydrogen bonds to or accepting dative bonds from solvent lone pairs in the case of acidic hydrogens or metal centres. Where this occurs, some covalent character and charge transfer character is expected in the solute:solvent interaction, and a large interaction energy is expected. The hydrogen bond between a fluoride ion and a water molecule leads to an interaction energy of $23 \mathrm{kcal} \mathrm{mol-1}$ in the gas phase 34 - definitely more than one might expect for a 'weak' bond. In such circumstances, it is not surprising that the perturbative approach embodied in continuum models is not very accurate.

The consequence of such strong interactions and their relatively poor description by continuum models is that calculated Gibbs energies of the affected species relative to those of less polar species are not always accurate. Apparently, with the typical parameterization of continuum models, the solvation Gibbs energies predicted by the models for ions tend to be insufficiently negative.35 A typical example of this is the calculation of acidity constants.36 For the acid dissociation of a species such as formic acid in water, one might hope to capture the equilibrium constant and hence the $\mathrm{p} K_{\mathrm{A}}$ by computing the Gibbs energy change for the reaction $\mathrm{HCOOH}+$ $\mathrm{H}_{2} \mathrm{O}<==>\mathrm{HCOO}_{-}+\mathrm{H}_{3} \mathrm{O}_{+}$(treating the proton as a naked $\mathrm{H}_{+}$combined with a continuum model would lead to an even more extreme example of the problem discussed here). However, with typical continuum models, this leads to a large overestimate of the Gibbs energy of the ions, hence 
to an underestimate of the equilibrium constant, and a predicted $\mathrm{p} K \mathrm{~A}$ that is larger than in experiment.

These problems with understabilization of high charge/radius ratio species can often be significantly removed by treating the solvent in a hybrid way, with several explicit solvent molecules included in the QM system, surrounded by the continuum.37 It has been suggested that the resulting Gibbs energies can be treated 'variationally', with the value of $n$ that returns the lowest Gibbs energy for the explicit solvation 'reaction' of the anion or cation X by solvent (here water) $\mathrm{X}_{(\text {aq })+/-}+n \mathrm{H}_{2} \mathrm{O}_{(\text {aq })}<==>\left(\mathrm{X}_{+/ .}\left(\mathrm{H}_{2} \mathrm{O}\right)_{n}\right)$ aq being assumed to also return the best estimate for the Gibbs energy of the solvated $\mathrm{X}$ ion.38 In this reaction, all species should be surrounded by continuum, and the appropriate standard state for the solvent should be used (e.g. for $\mathrm{H}_{2} \mathrm{O}, c_{0}=$ 55.5 M, so the Gibbs energy returned by standard quantum chemical codes based on a standard state of 1 bar $=p / R T=0.0409 \mathrm{M}$ should be corrected by a term $R T \ln (55.5 / 0.0409)=4.26$ $\mathrm{kcal} / \mathrm{mol}$ ). With this correction, and assuming that the continuum model yields exact results, and that all relevant conformers of $\mathrm{X}$ and its solvate have been included, then the Gibbs energy change for the solvation 'reaction' mentioned above would be zero for all $n$. In practice, this is often found not to be the case, and this signals the need for inclusion of some explicit solvent molecules together with the continuum. With smaller values of $n$ and when interactions with the solvent occur, it is usually possible to find all relevant conformers of the solvate complex $\mathrm{X}+/ . .\left(\mathrm{H}_{2} \mathrm{O}\right)_{\mathrm{n}}$, but great care should be taken with this aspect, and in practice the use of hybrid solvation models is probably best restricted to small numbers $n$ of explicit solvent molecules, modelling only those whose positions are quite rigidly enforced by their interaction with the solute.

Occasionally, organic and organometallic reactivity relevant for homogeneous catalysis is studied in conjunction with fully explicit solvent models, using either methods such as molecular 
mechanics,19 hybrid quantum mechanics/molecular mechanics (QM/MM) or ab initio direct dynamics in its various flavours.20 For example, this approach has been used to examine the relative Gibbs energies associated with the Schlenk equilibrium of Grignard reagents. 39 Explicit treatment of a large number of solvent molecules is in principle a very attractive way to treat solvation, since the latter is now 'correctly' described at the microscopic level. There is no space to describe this family of techniques in detail, but we wish to point out that such methods typically have high computational expense, which in turn leads to compromises concerning the accuracy of the potential energy method used. These methods also require extensive sampling in order to return reliable structural or energetic results, as the familiar rigid-rotor harmonic oscillator approaches to computing Gibbs energies become impossible to apply in this case. Great care is also needed with ionic solutes in order to obtain a good treatment of electrostatics at long range. Therefore, these techniques are by no means guaranteed to be more accurate than the much easier to perform QM + continuum solvent approaches, or even to be of equal quality to them, and are perhaps best reserved for special cases.

\section{Microscopic / Structural Models}

When modelling homogeneous catalysis using quantum chemical methods, one very important factor is the choice of the microscopic model used to perform the calculations, and to calculate relative energies. In the early days of quantum chemical studies of reactivity, the necessity of making difficult choices when model building was obvious, as computational restrictions usually made it impossible to study the 'real' reactive system. For example, as late as the 1990 s there was considerable discussion concerning whether $\mathrm{PMe}_{3}$ or $\mathrm{PH}_{3}$ was a 'better' model for the triphenylphosphine ligand. With the huge progress in computer speeds and computational 
methods, this aspect is nowadays perhaps less obvious, but it remains very important, and is a potential source of severe error. The issue arises in a variety of ways.

One of these arises when small molecules or ions that are gained or lost by the reacting system during a particular reaction step. The simplest example of such a fragment is the proton, but others possibilities are the bases involved in $\mathrm{C}-\mathrm{H}$ bond activation reaction mechanisms, species such as zinc oxide formed as a by-product in reactions of organozinc compounds, or counterions. When a modelled reaction mechanism involves uptake of a proton, calculated relative Gibbs energies will vary enormously depending on which starting species is used. Consider for example a reaction taking place in methanol, in which a species B can react to form products either in its original form, or after addition of a proton to yield $\mathrm{BH}_{+}$. Should this protonation be modelled as $\mathrm{B}+\mathrm{H}_{+} \rightarrow$ $\mathrm{BH}+$ or as $\mathrm{B}+\mathrm{MeOH} \rightarrow \mathrm{BH}_{+}+\mathrm{MeO}_{-}$or as $\mathrm{B}+\mathrm{HA} \rightarrow \mathrm{BH}_{+}+\mathrm{A}_{-}^{-}$, where $\mathrm{HA}$ is some acidic species known to be present in the reaction system? The choice will lead to very different relative Gibbs energies for $\mathrm{B}$ and $\mathrm{BH}+$, which may well affect very strongly the predicted mechanism.

The proton is an extreme example, and while it was not uncommon some years back to see studies in which 'acid catalysis' was casually argued for based on the observation that the addition of a proton led to a decrease in Gibbs energy, such gross errors are seldom made nowadays. Still, the inclusion of the thermodynamics of the originating acid and thereby the balanced computation of the relative Gibbs energy of $\mathrm{B}$ and $\mathrm{BH}+$ and hence of the ensuing transition states is not always correctly handled. What needs to be done here is to look carefully at the experimental conditions one is seeking to model, and to identify which species will be the proton donor under those conditions, and thereby compute the Gibbs energy change (and perhaps the barrier) for the reaction $\mathrm{B}+\mathrm{HA} \rightarrow \mathrm{BH}_{+}+\mathrm{A}_{-}$. 
Similar issues arise for other (small) molecules. Consider hydroboration: the reagent can be written in shorthand as $\mathrm{BH}_{3}$, but a correct treatment of Gibbs energies in ether solvent will require modelling it as an etherate complex. Or consider a reaction leading ultimately to formation of solid zinc oxide. How should this product be modelled? Quantum chemists may think of modelling it as the diatomic molecule $\mathrm{ZnO}$, or as a cluster $\mathrm{Zn}_{\mathrm{x}} \mathrm{O}_{\mathrm{x}}$, or perhaps think of using periodic quantum chemical methods to treat the solid. The first two options are not expected to be very accurate, and the periodic option leads to difficulties in comparing calculated energies obtained with molecular quantum chemical methods. If one only needs to model the relative Gibbs energy of this product, and not its kinetics of formation, a useful trick here can be to combine experimental data for the formation of gas-phase $\mathrm{Zn}$ and $\mathrm{O}$ atoms from solid $\mathrm{ZnO}$, with computational data concerning the relative Gibbs energy of gas-phase $\mathrm{Zn}$ and $\mathrm{O}$ atoms.40 Another example is the relative Gibbs energy of $\mathrm{Co}_{2}(\mathrm{CO})_{8}$ compared to $\mathrm{HCo}(\mathrm{CO})_{4}$ in presence of $\mathrm{H}_{2}$ during hydroformylation catalysis. 41 While the relative Gibbs energy of species containing a single cobalt atom could be predicted accurately using coupled-cluster methods, the dimeric species was less well described. However, experimental data for the equilibrium $\mathrm{Co} 2(\mathrm{CO})_{8}+\mathrm{H}_{2}<==>2 \mathrm{HCo}(\mathrm{CO})_{4}$ is available and could be used to supplement the computational data.41

A related issue concerning microscopic models arises when considering catalysts that can exist in a large number of different aggregation states, some of which are active, while others are not. If the inactive form is more stable than the active form, neglecting the aggregation in a computational study can lead to incorrect predictions of reactivity.42,43 As mentioned in the previous section, inclusion of some solvent molecules can also be important for obtaining accurate results. Finally, another problem of this type occurs when treating ionic species reacting in non-polar solvents, where they are usually present as ion pairs. Including the counterion may be important when 
studying reactivity, as its presence can modify the predicted reactivity. 44 The key to accurate models in all such cases is careful reflection on (and/or computational investigation of) the likely microscopic composition of the relevant species under the target experimental conditions.

\section{Conformational complexity}

In the early days of computational mechanistic studies, the models were rather simple and small enough that the modeller could easily ensure that the correct conformation was obtained by the geometry optimization procedure. Today, however, models have reached a size such that conformational complexity has become an issue one needs to pay careful attention to. The error bar associated to conformer choice has been shown to be as large as $10 \mathrm{kcal} \mathrm{mol-1}$ for species involving bulky ligands such as that shown in Figure 1.45 In general, one has to make sure that the located stationary points on the energy profile (intermediates and transition states) correspond to the desired structure for the groups that participate in the reaction event, and to the global minimum for the parts that do not directly participate. Experimental data as well as computation can help to ensure that this has been done.46 Failure to do this can have severe consequences on the mechanistic conclusions, in particular when it comes to the analysis of selectivity (vide infra), and the literature is unfortunately full of cases where incorrect mechanisms were advocated on the basis of wrong energy minima. 

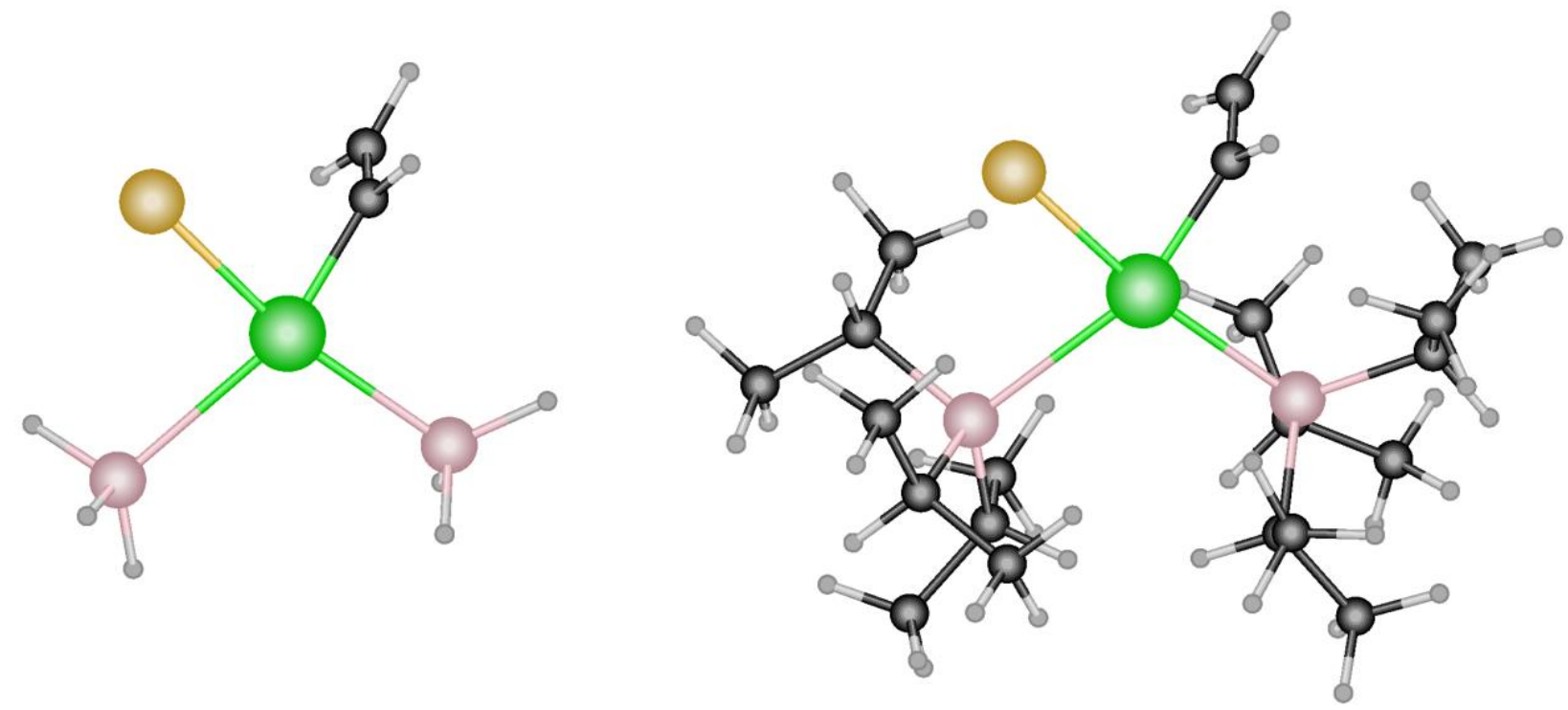

Figure 1. Example of a small (left) and large (right) model of an organometallic complex. The larger model has a much more varied conformational landscape.

Conformational analysis is today mainly performed manually. That is, a large number of possible starting conformations (e.g. isomers and rotamers) are generated explicitly by the user and the geometries are then optimized and their energies compared. This usually means at least tens of starting structures for each stationary point, which leads to very tedious and time-consuming work. Also, as the system becomes larger, the conformational space grows (exponentially with the number of atoms47) so the risk of missing the global minimum becomes more serious, even for experienced computational chemists. Some groups use efficient algorithms for exploring the conformational space and identifying the global minimum at the molecular mechanics level, prior to refinement with quantum chemical methods, 48 though this only works if the structure and relative energy of the different conformers are at least reasonably well reproduced by the low-level method. In the context of reactivity, more systematic techniques for automatic exploration of 
potential energy surfaces are very promising for finding relevant minima and transition states 49 but their computational demand remains currently high for realistic organometallic system.

As a final comment about conformational sampling, we wish to mention that in most cases, the density of states within the conformational manifold is not inordinately high in the sort of models typically studied in computational homogeneous catalysis. It is therefore normally sufficient to use the lowest-energy conformation of each species when computing Gibbs energies for use in the mechanistic analysis. Boltzmann averaging over multiple conformers so as to include configurational entropy is often not needed, as the lowest-energy form will often dominate the distribution. There are however examples involving long alkyl chains interacting with organic fragments where extended number of conformations have to be applied. In a study on osmiumcatalyzed dihydroxylation of 1-decene it was found that the lowest energy transition state accounted for only $10 \%$ the total yield.50 


\section{Kinetic modelling}

Catalysis is an intrinsically kinetic effect, so computational studies of homogeneous catalysis are of necessity concerned with kinetics. A frequent aim of such studies is to establish the mechanism of the catalysed process, or to elucidate the nature of the steps that lead to selectivity of one kind or another. How does one relate the computed potential energy surface to reactivity? The first part of the answer is that one can compute rate constants for individual elementary steps by using transition state theory (TST). In most cases, one uses canonical TST, usually in the form of the Eyring equation, whereby the rate constant $k$ can be expressed from the Boltzmann and Planck constants $k$ в and $h$, as a function of the temperature $T$ and the activation Gibbs energy $\Delta G \ddagger$ for the corresponding step, $k=k \mathrm{~B} T / h \exp (-\Delta G \ddagger / R T$ ) (for bimolecular reaction steps, one should strictly speaking include the corresponding standard state concentration in the denominator of this expression). For accurate results, it can be necessary to include symmetry numbers.51 For individual elementary steps, then, a direct link between calculated Gibbs energies and experimental rate constants can be made. While TST is not an exact theory (see below), the evidence from gas-phase studies is that it often yields results that are accurate to within an order of magnitude.52 An error on rate constants by a factor of ten at room temperature is equivalent to an error on the activation Gibbs energy by about $1.4 \mathrm{kcal}$ mol-1. Obtaining this level of accuracy in the quantum chemistry component of a study is far from straightforward, so in comparison, TST can provisionally at least be assumed to lead to useful accuracy. Most experienced computational chemists will be adept at mapping a calculated activation Gibbs energy for a simple process onto a predicted reactivity.

But not all cases are straightforward. A first problem arises when trying to identify the key species defining the 'rate' of the catalytic cycle. Experimental chemists often talk about the rate 
determining step, and computational chemists sometimes interpret this as being the elementary step within the mechanism with the highest (Gibbs) energy barrier. This is however an oversimplification, as shown e.g. by the energetic span model.53 This model, whose use should be mandatory in any analysis of computed Gibbs energy profiles in catalysis, identifies a 'rate determining intermediate' and a 'rate determining transition state', which need not be consecutive. From these two structures, the overall barrier for the catalytic cycle can be computed in most cases. The energetic span model preserves the highly intuitive Gibbs energy surface model emerging from the computations, while providing a refined analysis of the emerging reactivity.

There are still situations however where a qualitatively valid analysis requires the explicit introduction of concentration effects. There are at least two general cases where this has been identified to be necessary: complicated reaction networks 54,55 and presence of highly different concentrations in competing steps.41 The most reliable way to extract the predicted kinetic behaviour from a computed Gibbs energy surface is to carry out explicit modelling of the kinetics.56 This can be done in a variety of ways, with the most simple being similar to those used in analysis of the kinetics of mechanisms carried out in conventional experimental work.57 However, this approach suffers from the problems identified above in the case of complicated mechanisms, and it is in many ways preferable to carry out explicit numerical integration of the kinetic equations after inputting appropriate initial concentrations for the reactants and catalysts. Powerful algorithms are available for ordinary differential equation integration, and are indeed available in a number of software packages that can be directly used for simulating catalytic kinetics.

When performing such modelling, the typical procedure is to identify all relevant reactants, intermediates, products, and transition states, set the initial concentrations, and then model the 
kinetics. The outcome is usually a time-series for the concentrations of each species, which can be plotted graphically (see Fig 2). Reaction fluxes are obtained for each reaction path, and detailed analysis can then provide insight into the location of the reaction bottleneck. Further insight is available by integration of the kinetics several times with changes in the initial concentration. If desired, based on an analysis of the fluxes, one may be able to deduce a reduced set of reactions that are sufficient to reproduce the observed kinetics. Together with the steady-state approximation, this can also be used in order to extract an empirical rate law, thereby facilitating comparison to experiment.

It should be noted that such a model usually requires quite a lot of rate constants, for both the forward and reverse reactions corresponding to many different TSs. The required steps can be most easily identified by drawing a map of species and reaction steps, as is common in experimental analyses of reaction mechanisms. 


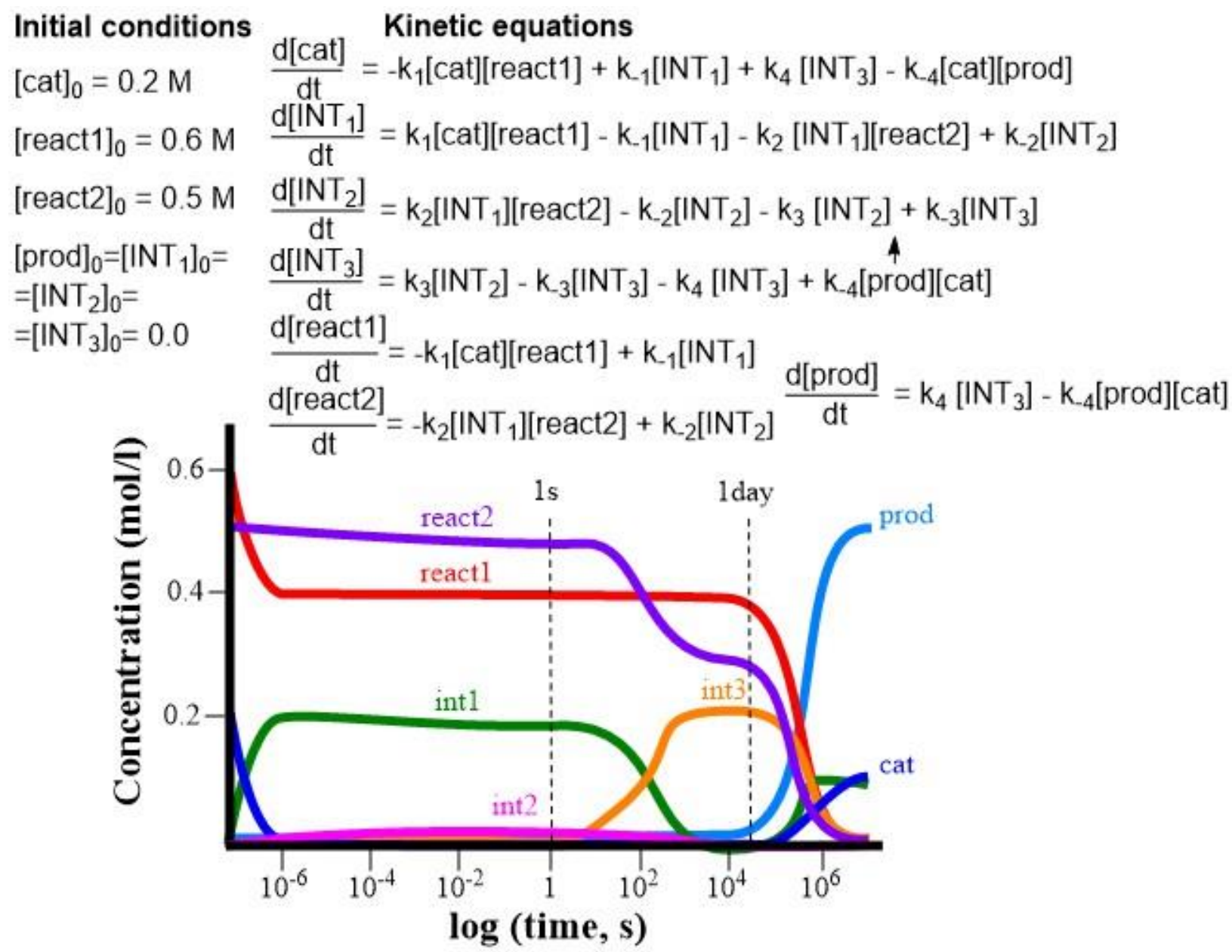

Figure 2: Set-up of kinetic equations (top) and resulting concentation-time series (bottom) for a kinetic model

A final point to be made in relation to kinetics and homogeneous catalysis relates to barrierless reactions. By this we mean reactions in which species $\mathrm{A}$ and $\mathrm{B}$ can react to form $\mathrm{C}$ without needing to cross a potential energy barrier. They may not be critical when evaluating the viability of a reaction mechanism, as they correspond to fast steps, but a rate constant value is nevertheless required in order to build a kinetic model, and such steps can affect the overall predicted rate of turnover.41 They occur for a wide range of bimolecular reaction steps common in catalysis, such 
as addition of some radicals to other species, or addition of a ligand to a coordinatively unsaturated metal centre. Careful scanning of the potential energy surface often shows that in such cases there is no barrier or saddle-point. In the absence of a saddle-point on the potential energy surface (or on the potential of mean force including Gibbs energy contributions from the continuum solvent model), it is not possible to use the standard harmonic frequency analysis needed to provide input for statistical mechanics and thereby for application of TST. More sophisticated TST variants can be used in such cases, but we have found that good results can also be obtained by assuming that such steps occur in the diffusion-limited regime. Based on a combination of theories of diffusion and of collisions, 58 a simple expression for the rate constant can then be obtained with as only needed input the solvent viscosity $\eta$ and the temperature $T: k=8 k \mathrm{~B} T / 3 \eta$. With typical solvent viscosities near room temperature, this corresponds to the magnitude of rate constant that would arise from the Eyring equation with a Gibbs energy barrier of roughly 4 kcal mol-1.

\section{Challenges Associated with Selectivity}

The study of selectivity poses additional challenges to methodology due to the large effect of small differences in energy. Gibbs energy differences between transition states can be re-expressed as product ratios through simple application of Boltzmann weighting, providing a ready means to test agreement with experiment.59,60 This agreement is often assessed more qualitatively, with the correct sign for the key difference being interpreted as agreement with experiment, even though the underlying Gibbs energies map poorly onto the observed outcome. It is for instance regrettable to find publications where a 9:1 preference for a given product (e.g. an enantiomeric excess of $80 \%$ ) is justified with a calculation indicating a difference of more than $10 \mathrm{kcal}$ mol-1 between the competing transition states. At room temperature, relative rates of 9:1 arise from Gibbs energy 
barrier differences of $1.3 \mathrm{kcal}$ mol-1. From this it follows that if an error of a few kcal mol-1 can be tolerated in mechanistic studies, the accuracy required to investigate selectivity should be much higher than that, on the order of less than $1 \mathrm{kcal} \mathrm{mol-1}$ in relative transition state energy. This might sound unachievable, but fortunately competing selectivity-determining transition states are commonly quite similar in structure and many of the systematic errors will therefore cancel when considering their relative Gibbs energy. Here, the problem of conformational search becomes particularly significant, and one has to pay special attention to the geometry of the selectivitydetermining TS.

Calculations have in recent years been very successful in solving problems concerned with various kinds of selectivity, such as chemo-, regio- and enantioselectivity. A large number of outstanding questions has been addressed and a lot of insight has been gained. Enantioselectivity deserves a special mention here. In many systems, discrimination between enantiomers is dictated by steric interactions, and as these usually are associated with weak interactions, dispersion effects have to be included in the calculations.61

As an example, one can mention the study on the tetrapeptide-catalyzed kinetic resolution of trans-2-N-acetamidocyclohexanol.62 Although the peptide structure is constrained by internal hydrogen bonds, a large number of conformeric and diastereoisomeric transition states $(>50)$ of the selectivity-determining step had to be located in order to pinpoint the origins of the observed enantioselectivity (see Fig. 3). Here, inclusion of dispersion correction was critical in order to reproduce the experimental outcome. 


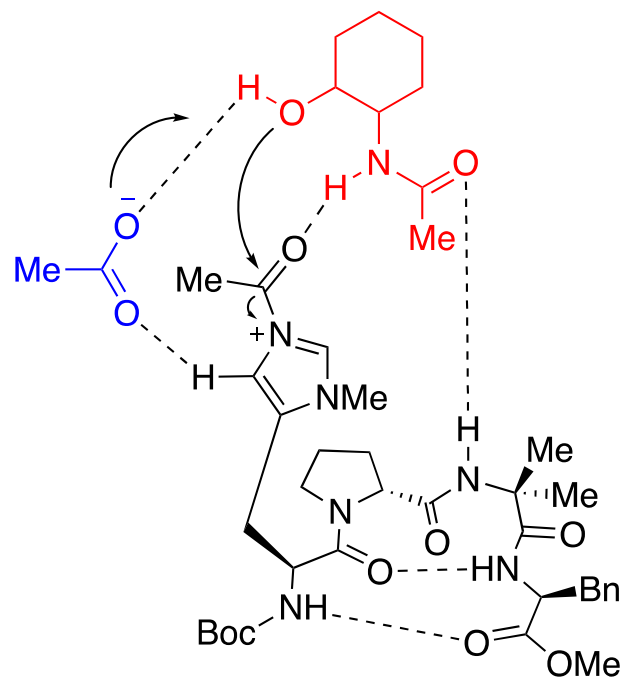

Figure 3. Selectivity-determining transition state for the tetrapeptide-catalyzed kinetic resolution of trans-2-N-acetamidocyclohexanol.

\section{Additional Aspects}

The expansion of computational homogeneous catalysis means that it now encompasses many topics that were seldom considered before. These aspects cannot all be mentioned here, nor can any of them be explained in detail, but it is useful to at least highlight the fact that depending on the problem at hand, there may be a need to expand the theoretical framework considerably.

A previous section discussed the use of kinetics modelling to understand reaction in homogeneous catalysis, and made frequent reference to the Eyring equation of transition state theory. It was implied that provided that the correct Gibbs energies for the reactant and transition states were used, this theory provides the correct rate constant. This is, however, certainly not always the case, because TST is an approximate theory. The exact theory that should be applied to describe chemical reactivity on potential energy surfaces is quantum mechanics, and both timeindependent and time-dependent versions of this theory for describing the motion of atoms in 
chemical reactions are available. However, these theories are extremely demanding to perform calculations with, and have by and large been limited to describing reactions of small molecules in the gas phase. Together with accurate potential energy surfaces, they provide very good agreement with experiment. Also, their predictions differ from those of TST due to factors such as tunnelling, wavepacket 'diffraction' and thereby interference through the TS region, and nonthermal energy distributions for intermediates. In many cases, especially for heavier nuclei or when including simple corrections for tunnelling effects for protons, TST can in fact produce results that agree quite well with quantum dynamics, but in other cases, severe differences are obtained. Although quantum dynamics may be desirable, they are impractical for most applications in computational homogeneous catalysis.

Classical mechanics, in the form of Newtonian trajectory studies, can also be used to study dynamics of chemical reactions. Provided the potential energy surface can be computed at the large number of points needed, classical trajectories are relatively inexpensive to compute and the expense scales well with the size of the system. Hence such trajectory studies have been quite commonly performed for reactive systems relevant to homogeneous catalysis, including in some cases the presence of solvent. The performance of molecular dynamics seems to have an important added value in reactions in protic solvents, in particular cases involving stabilization of nascent charged fragments via microscopic solvation, transfer of protons between distant fragments mediated by solvent molecules, and solvent coordination to unsaturated metal centres.63,64

One example of an important dynamic effect occurs in multi-step reaction mechanisms, where an exothermic step is followed by branching between multiple possible reaction steps, each involving relatively low Gibbs energy barriers. In such cases, the intermediate formed from the prior step will initially be quite 'hot' in terms of internal ro-vibrational energy, and thermal 
equilibration with solvent will occur only on a timescale of the order of multiple picoseconds.65 If this is also the timescale for the subsequent reactions, then the assumption that the branching ratio between these steps can be determined using the Eyring equation of TST and the overall bulk temperature may be incorrect.66 A variant of such a problem can occur when the energy redistribution needs to occur intra-molecularly.67 The correct treatment of such problems will require energy-resolved statistical rate theories or indeed dynamical methods.

Another area that is gaining importance is the study of single electron transfer processes (SET). Inner-sphere electron transfer is tractable with standard approaches, although sometimes broken symmetry solutions have to be forced into DFT. The treatment of outer-sphere electron transfer is more complex, and some success has been reached with the use of Marcus theory.68,69,70

Another aspect that will become increasingly important as computational chemists make more and more contributions to experimental studies of catalysis is aspects relating to chemical process technology. The change of phase of some reactants and products, the type of reactor (e.g, batch, semi-batch, continuous), the type of stirring and the efficiency of heat regulation can all have a significant influence on the outcome of a chemical reaction. These aspects are commonly addressed in chemical engineering modelling, and the tools to address these factors in enhanced kinetics modelling are therefore available.

\section{Conclusions}

In this perspective, we have tried to give an overview of some of the main areas relating to the methodology of computational studies of homogeneous catalysis which we feel are important for obtaining the most reliable results possible, and which go beyond the aspect of electronic structure theory. Computational work remains challenging, and even the most careful work will not 
necessarily yield exact results, but careful design of the computational protocol and of the project goals can play a role in making the results obtained more meaningful. Developments of understanding and methodology in these areas continue to occur, partly due to progress in the electronic structure methods. As a result, some of the approaches that we recommend in the present perspective were not widely in use just a few years ago, including in work from our own groups. We believe that since these other aspects are each able to influence computed relative energies by many kcal mol-1, or to impact on the predicted outcome of chemical reactions in terms of their selectivity or overall rate by at least one and often several orders of magnitude, they are of great importance and should be taken into account when designing or analyzing any computational study in the field.

\section{AUTHOR INFORMATION}

\section{Corresponding Author}

* All authors may be contacted for information concerning this work.

\section{Author Contributions}

The manuscript was written through contributions of all authors. All authors have given approval to the final version of the manuscript.

\section{ACKNOWLEDGMENT}

JNH thanks KU Leuven for funding through grant C14/15/052. The authors thank Oriana Brea for help with figures. We also acknowledge co-workers and colleagues for many helpful 
comments and suggestions over the years. We specifically wish to thank our experimental

colleagues and collaborators for their critical engagement with computational work.

\section{REFERENCES}

1 Noyori, R.; Richmond, J. P. Ethical Conduct in Chemical Research and Publishing. Adv. Synth. Catal. 2013, 355, 38.

2 Plata, R. E.; Singleton, D. A. A Case Study of the Mechanism of Alcohol-Mediated Morita Baylis-Hillman Reactions. The Importance of Experimental Observations. J. Am. Chem. Soc. 2015, 137, 3811-3826.

3 Grimme, S.; Antony, J.; Ehrlich, S.; Krieg, H. A Consistent and Accurate Ab Initio Parametrization of Density Functional Dispersion Correction (DFT-D) for the 94 Elements H-Pu. J. Chem. Phys. 2010, 132, 154104.

4 Hättig, C.; Klopper, W.; Köhn, A.; Tew, D. P. Explicitly Correlated Electrons in Molecules. Chem. Rev. 2012, 112, 4-74.

5 Riplinger, C.; Neese, F. An Efficient and Near Linear Scaling Pair Natural Orbital Based Local Coupled Cluster Method. J. Chem. Phys. 2013, 138, 034106; Werner, H.-J.; Schütz, M. An Efficient Local Coupled Cluster Method for Accurate Thermochemistry of Large Systems. J. Chem. Phys. 2011, 135, 144116.

6 Ufimtsev, I. S.; Martinez, T. J. Quantum Chemistry on Graphical Processing Units. 1. Strategies for Two-Electron Integral Evaluation. J. Chem. Theory Comput. 2008, 4, 222-231.

7 Tsang, A. S. L.; Sanhueza, I. A.; Schoenebeck, F. Combining Experimental and Computational Studies to Understand and Predict Reactivities of Relevance to Homogeneous Catalysis, Chem. Eur. J. 2014, 20, 16432-16441.

8 Santoro, S.; Kalek, M.; Huang, G.; Himo, F. Elucidation of Mechanisms and Selectivities of Metal-Catalyzed Reactions using Quantum Chemical Methodology, Acc. Chem. Res. 2016, 49, 1006-1018.

9 Davies, D. L.; Macgregor, S. A.; McMullin, C. L. Computational Studies of Carboxylate-Assisted C-H Activation and Functionalization at Group 8-10 Transition Metal Centers. Chem. Rev. 2017, 117, 8649-8709.

10 Eisenstein, O.; Milani, J.; Perutz, R. N. Selectivity of C-H Activation and Competition between C-H and C-F Bond Activation at Fluorocarbons. Chem. Rev. 2017, 117, 8710-8753.

11 Yang, Y.-F.; Hong, X.; Yu, J.-Q.; Houk, K. N. Experimental Computational Synergy for Selective Pd(II)-Catalyzed C-H Activation of Aryl and Alkyl Groups. Acc. Chem. Res. 2017, 50, 2853-2860.

12 Maji, R.; Mallojjalla, S. C. ; Wheeler, S. E. Chiral Phosphoric Acid Catalysis: From Numbers to Insights. Chem. Soc. Rev. 2018, 47, 1142-1158.

13 Odoh, S. O.; Cramer, C. J.; Truhlar, D. G.; Gagliardi, L. Quantum-Chemical Characterization of the Properties and Reactivities of Metal-Organic Frameworks. Chem. Rev. 2015, 115, 6051-6111.

14 Jover, J.; Fey, N. The Computational Road to Better Catalysts. Chem. Asian J. 2014, 9, 1714-1723.

15 Hopmann, K. How Accurate is DFT for Iridium-Mediated Chemistry? Organometallics 2016, 35, $3795-3807$.

16 Kitaura, K.; Obara, S.; Morokuma, K. Transition State of Oxidative Addition Reaction: $\mathrm{Pt}_{(}\left(\mathrm{PH}_{3}\right) 2+\mathrm{H}_{2} \rightarrow$ $\mathrm{Pt}(\mathrm{H})_{2}\left(\mathrm{PH}_{3}\right)_{2}$. J. Am. Chem. Soc. 1981, 103, 2891-2892.

17 Pulay, P. Localizability of Dynamic Electron Correlation. Chem. Phys. Lett. 1983, 100, 151-154.

18 Watson, L. A.; Eisenstein, O. Entropy Explained: The Origin of Some Simple Trends. J. Chem. Ed. 2002, 79, 12691277.

${ }_{19}$ Liu, Z.; Patel, C.; Harvey, J. N.; R. B. Sunoj, R. B. Mechanism and Reactivity in the Morita-Baylis-Hillman Reaction: the Challenge of Accurate Computations. Phys. Chem., Chem. Phys., 2017, 19, 30647 - 30657.

20 Besora, M.; Vidossich, P.; Lledós, A.; Ujaque, G.; Maseras, F. Calculation of Reaction Free Energies in Solution: A Comparison of Current Approaches. J. Phys. Chem. A 2018, 122, 1392-1399.

${ }_{21}$ Grimme, S. Supramolecular Binding Thermodynamics by Dispersion-Corrected Density Functional Theory. Chem. Eur. J. 2012, 18, $9955-9964$

22 Marenich, A. V.; Olson, R. M.; Kelly, C. P.; Cramer, C. J.; Truhlar, D. G. Self-Consistent Reaction Field Model for Aqueous and Nonaqueous Solutions Based on Accurate Polarized Partial Charges. J. Chem. Theory Comput. 2007, 3 , 2011-2033. 
23 Piccini, G. M.; Sauer, J. Effect of Anharmonicity on Adsorption Thermodynamics. J. Chem. Theory Comput. 2014, 10, 2479-2487.

24 Abraham, M. H. Relationship Between Solution Entropies and Gas Phase Entropies of Nonelectrolytes. J. Am. Chem. Soc. 1981, 103, 6742-6744.

25 e.g. Cooper, J.; Ziegler, T. A Density Functional Study of $\mathrm{S}_{\mathrm{N}} 2$ Substitution at Square-Planar Platinum(II) Complexes. Inorg. Chem. 2002, 41, 6614-6622; Lau, J. K. C.; Deubel, D. V. Hydrolysis of the Anticancer Drug Cisplatin: Pitfalls in the Interpretation of Quantum Chemical Calculations. J. Chem. Theory Comput. 2006, 2, 103106.

26 Mammen, M.; Shaknovich, E. I.; Deutch, J. M.; Whitesides, G. M. Estimating the Entropic Cost of Self-Assembly of Multiparticle Hydrogen-Bonded Aggregates Based on the Cyanuric Acid·Melamine Lattice. J. Org. Chem. 1998, $63,3821-3830$.

27 For a recent development of this type of approach, see Garza, A. J. Solvation Entropy Made Simple. J. Chem. Theory Comput. 2019, 15, 3204-3214.

28 Ribeiro, R. F.; Marenich, A. V.; Cramer, C. J.; Truhlar, D. G. Use of Solution-Phase Vibrational Frequencies in Continuum Models for the Free Energy of Solvation. J. Phys. Chem. B, 2011, 115, 14556-14562.

29 Santoro, S.; Kalek, M.; Huang, G.; Himo, F. Elucidation of Mechanisms and Selectivities of Metal-Catalyzed Reactions using Quantum Chemical Methodology, Acc. Chem. Res. 2016, 49, 1006-1018.

30 Reichardt, C. Solvents and Solvent Effects in Organic Chemistry, 3rd edition, Wiley-VCH, Weinheim, 2003.

31 Tomasi, J.; Mennucci, B. ; Cammi, R. Quantum Mechanical Continuum Solvation Models. Chem. Rev. 2005, 105, 2999-3093.

32 These two approaches usually yield very similar structures and relative free energies. Where the gradient of the Gibbs energy of solvation with respect to the coordinates of the atoms is large in the vicinity of the minimum, larger changes may be obtained, and care will be needed to assess how reliably these charges are reproduced by the solvation model.

33 Minenkov, Y.; Occhipinti, G.; Jensen, V. R. Metal-Phosphine Bond Strengths of the Transition Metals: A Challenge for DFT. J. Phys. Chem. A 2009, 113, 11833-11844.

34 Arshadi, M.; Yamdagni, R.; Kebarle, P. Hydration of the Halide Negative Ions in the Gas Phase. II. Comparison of Hydration Energies for the Alkali Positive and Halide Negative Ions. J. Phys. Chem. 1970, 74, $1475-1482$.

35 Kelly, C. P.; Cramer, C. J.; Truhlar, D. G. Adding Explicit Solvent Molecules to Continuum Solvent Calculations for the Calculation of Aqueous Acid Dissociation Constants. J. Phys. Chem. A 2006, 110, 2493-2499.

36 Ho, J.; Coote, M. L. A Universal Approach for Continuum Solvent pKa Calculations: are We There Yet? Theor. Chem. Acc. 2010, 125, 3-21.

37 For an early systematic discussion of this approach, see Bryantsev, V. S.; Diallo, M. S.; Goddard, W. A., III. Calculation of Solvation Free Energies of Charged Solutes Using Mixed Cluster/Continuum Models. J. Phys. Chem. B 2008, 112, 9709-9719.

38 Pliego, J. R.; Riveros, J. M. The Cluster-Continuum Model for the Calculation of the Solvation Free Energy of Ionic Species. J. Phys. Chem. A 2001, 105, 7241-7247.

${ }_{39}$ Peltzer, R. M.; Eisenstein, O.; Nova, A.; Cascella, M. How Solvent Dynamics Controls the Schlenk Equilibrium of Grignard Reagents: A Computational Study of $\mathrm{CH}_{3} \mathrm{MgCl}$ in Tetrahydrofuran. J. Phys. Chem. B, 2017, 121, 42264237.

40 This procedure was used in Bedford, R. B.; Gower, N. J.; Haddow, M. F.; Harvey, J. N.; Nunn, J.; Okopie, R. A.; Sankey, R. F. Exploiting Boron-Zinc Transmetallation for the Arylation of Benzyl Halides: What are the Reactive Species? Angew. Chem., Int. Ed. 2012, 51, 5435-5438.

41 Rush, L. E.; Pringle, P. G.; Harvey, J. N. Computational Kinetics of Cobalt-Catalyzed Alkene Hydroformylation. Angew. Chem. Int. Ed. 2014, 53, 8672-8676.

42 Ribeiro, R.; Ruivo, R.; Nsiri, H.; Norsic, S.; D’Agosto, F.; Perrin, L.; Boisson, C. Deciphering the Mechanism of Coordinative Chain Transfer Polymerization of Ethylene using Neodymocene Catalysts and Dialkylmagnesium. ACS Catal. 2016 6, 851-860; Nsiri, H.; Belaid, I.; Larini, P.; Thuilliez, J.; Boisson, C.; Perrin, L. Ethylene-Butadiene Copolymerization by Neodymocene Complexes: a Ligand Structure / Activity / Polymer Microstructure Relationship Based on DFT calculations. ACS Catal. 2016 6, 1028-1036.

43 Zhang, J.; Szabó, K.J.; Himo, F. Metathesis Mechanism of Zinc-Catalyzed Fluorination of Alkenes with Hypervalent Fluoroiodine, ACS Catal. 2017, 7, 1093-1100; Huang, G.; Kalek, M.; Liao, R.-Z.; Himo, F. Mechanism, Reactivity, and Selectivity of the Iridium-Catalyzed C(sp3)-H Borylation of Chlorosilanes, Chem. Sci. 2015, 6, 17351746.

44 Kovacevic, A.; Gründemann, S.; Miecznikowski, J. R.; Clot, E.; Eisenstein, O.; Crabtree, R. H. Counter-ion Effects Switch Ligand Binding from C-2 to C-5 in Kinetic Carbenes Formed from an Imidazolium Salt and $\operatorname{IrH}_{5}\left(\mathrm{PPh}_{3}\right)_{2}$ Chem. 
Commun. 2002, 2580-2581; Appelhans, L. N.; Zuccaccia,D.; Kovacevic, A.; Chianese, A. R.; Miecznikowski, J. R.; Macchioni, A.; Clot, E.; Eisenstein, O.; Crabtree, R. H. An Anion-Dependent Switch in Selectivity Results from a Change of $\mathrm{C}-\mathrm{H}$ Activation Mechanism in the Reaction of an Imidazolium Salt with $\operatorname{IrH}_{5}\left(\mathrm{PPh}_{3}\right)_{2}$. J. Am. Chem. Soc. 2005, 127, 16299-16311.

${ }_{45}$ Besora, M.; Braga, A. A. C.; Ujaque, G.; Maseras, F.; Lledós, A. The Importance of Conformational Search: a Test Case on the Catalytic Cycle of the Suzuki-Miyaura Cross-Coupling. Theor. Chem. Acc. 2011, 128, 639-646.

46 See for example Balcells, D.; Clot, E.; Eisenstein, O.; Nova, A.; Perrin, L. Deciphering Selectivity in Organic Reactions: A Multifaceted Problem. Acc. Chem. Res. 2016 49, 1070-1078.

47 Wales, D. J. Energy Landscape, Cambridge University Press, Cambridge, 2003.

48 For an example, Burns, M.; Essafi, S.; Bame, J. R.; Bull, S. P.; Webster, M. P.; Balieu, S.; Dale, J. W.; Butts, C. P.; Harvey, J. N.; Aggarwal, V. K. Assembly-line Synthesis of Organic Molecules with Tailored Shapes. Nature 2014, $513,183-188$.

49 See e.g. (a) Maeda, S.; Ohno, K.; Morokuma K. Systematic Exploration of the Mechanism of Chemical Reactions: the Global Reaction Route Mapping (GRRM) Strategy Using the ADDF and AFIR methods. Phys. Chem. Chem. Phys. 2013, 15, 3683-3701; (b) Dewyer A. L.; Argüelles, A. J.; Zimmerman, P. M. Methods for Exploring Reaction Space in Molecular Systems. WIREs Comput. Mol. Sci. 2018, 8, e1354 .

50 Drudis-Solé, G.; Ujaque, G.; Maseras, F.; Lledós, A. A QM/MM Study of the Asymmetric Dihydroxylation of Terminal Aliphatic n-Alkenes with $\mathrm{OsO}_{4} \bullet(\mathrm{DHQD})_{2} \mathrm{PYDZ}$ : Enantioselectivity as a Function of Chain Length. Chem. Eur. J. 2005, 11, 1017-1029.

51 Fernández-Ramos, A.; Ellingson, B. A.; Meana-Pañeda, R.; Marques, J. M. C.; Truhlar, D. G. Symmetry Numbers and Chemical Reaction Rates. Theor. Chem. Accts. 2007, 118, 813-826; Gilson, M. K.; Irikura, K. K. Symmetry Numbers for Rigid, Flexible, and Fluxional Molecules: Theory and Applications. J. Phys. Chem. B 2010, 114, 1630416317.

52 For an example, see Petit, A. S.; Harvey, J. N. Atmospheric Hydrocarbon Activation by the Hydroxyl Radical: A Simple Yet Accurate Computational Protocol for Calculating Rate Coefficients. Phys. Chem., Chem. Phys. 2012, 14, $184-191$.

53 Kozuch, S.; Shaik, S. How to Conceptualize Catalytic Cycles? The Energetic Span Model. Acc. Chem. Res. 2011, 44, 101-110.

54 Goehry, C.; Besora, M.; Maseras, F. Computational Study on the Mechanism of the Acceleration of 1,3-Dipolar Cycloaddition Inside Cucurbit[6]uril. ACS Catalysis 2015, 5, 2445-2451.

${ }_{55}$ Kalek, M.; Himo, F. Mechanism and Selectivity of Cooperatively Catalyzed Meyer-Schuster Rearrangement/TsujiTrost Allylic Substitution. Evaluation of Synergistic Catalysis by Means of Combined DFT and Kinetics Simulations. J. Am. Chem. Soc. 2017, 139, 10250-10266.

56 Besora, M.; Maseras, F. Microkinetic Modeling in Homogeneous Catalysis. WIREs Comput. Mol. Sci. 2018, 8, e1372.

57 See e.g. Song, L.; Zhang, X.; Tian, G.; Robeyns, K.; Van Meervelt, L.; Harvey, J. N.; Van der Eycken E. V. Intramolecular Cascade Annulation Triggered by $\mathrm{CH}$ Activation via Rhodium Hydride Intermediate. Molec. Catal. 2019, 463, 30-36.

${ }_{58}$ For a discussion, see K. J. Laidler, Chemical Kinetics, 3rd ed., Harper \& Row, New York, 1987.

59 Balcells, D.; Maseras, F. Computational Approaches to Asymmetric Synthesis. New J. Chem. 2007, 31, 333-343.

60 Nova, A.; Maseras, F. Enantioselective Synthesis. In Comprehensive Inorganic Chemistry II, 2nd ed.; Reedijk, J.;

Poeppelmeier, K., Eds.; Elsevier: Amsterdam, The Netherlands, 2013; Vol 9, pp 807-831.

61 Nordin, M.; Liao, R.-Z.; Ahlford, K.; Adolfsson, H.; Himo, F. Theoretical Study of Asymmetric Transfer Hydrogenation of Ketones Catalyzed by Amino Acid-Derived Rhodium Complexes, ChemCatChem 2012, 4, 10951104. Santoro, S.; Liao, R.-Z.; Marcelli, T.; Hammar, P.; Himo, F. Theoretical Study of Mechanism and Stereoselectivity of Catalytic Kinugasa Reaction, J. Org. Chem. 2015, 80, 2649-2660.

${ }_{62}$ Liao, R.-Z.; Santoro, S.; Gotsev, M.; Marcelli, T.; Himo, F. Origins of Stereoselectivity in Peptide-Catalyzed Kinetic Resolution of Alcohols. ACS Catal. 2016, 6, 1165-1171.

63 Vidossich, P.; Lledós, A.; Ujaque, G. First-Principles Molecular Dynamics Studies of Organometallic Complexes and Homogeneous Catalytic Processes. Acc. Chem. Res. 2016, 49, 1271-1278.

${ }_{64}$ Govindarajan, N.; Tiwari, A.; Ensing, B.; Meijer, E. J. Impact of the Ligand Flexibility and Solvent on the O-O Bond Formation Step in a Highly Active Ruthenium Water Oxidation Catalyst. Inorg. Chem. 2018, 57, 13063-13066. 65 For a discussion, see Essafi, S.; Harvey, J. N. Rates of Molecular Vibrational Energy Transfer in Organic Solutions. J. Phys. Chem. A, 2018, 122, 3535-3540.

${ }_{66}$ Carpenter, B. K.; Harvey, J. N.; Orr-Ewing, A. J. The Study of Reactive Intermediates in Condensed Phases. J. Am. Chem. Soc., 2016, 138, 4695-4795. 
67 Rabinovitch, B. S.; Rynbrandt, J. D. Intramolecular Energy Relaxation. Nonrandom Decomposition of Hexafluorobicyclopropyl. J. Phys. Chem. 1971, 75, 2164-2171; Bailey, J. O.; Singleton, D. A. Failure and Redemption of Statistical and Nonstatistical Rate Theories in the Hydroboration of Alkenes. J. Am. Chem. Soc. 2017, 44, 15710-15723.

68 Qi, Z.-H.; Ma, J. Dual Role of a Photocatalyst: Generation of Ni(0) Catalyst and Promotion of Catalytic C-N Bond Formation. ACS Catal. 2018, 8, 1456-1463.

69 de Aguirre, A.; Funes-Ardoiz, I.; Maseras, F. Four Oxidation States in a Single Photoredox Ni-Based Catalytic Cycle: A Computational Study. Angew. Chem. Int. Ed. 2019, 58, 3898-3902.

70 Bonet, A.; Odachowski, M.; Leonori, D.; Essafi, S.; Aggarwal, V. K. Enantiospecific sp2-sp3 Coupling of Secondary and Tertiary Boronic Esters. Nature Chem. 2014, 6, 584-589. 